\title{
Raloxifene Protects Cisplatin-Induced Renal Injury in Mice via Inhibiting Oxidative Stress
}

\author{
Jian-Hong An $\mathbb{D}^{\prime}$ \\ Chun-Yan Li (D) \\ Chun-Ya Chen (iD) \\ Jian-Bin $\mathrm{Wu}^{2}$ \\ Hong Shen $\mathbb{D}^{1,3}$ \\ 'School of Medicine, South China \\ University of Technology, Guangzhou \\ Higher Education Mega Center, \\ Guangzhou, 5I 0006, People's Republic of \\ China; ${ }^{2}$ Department of Oncology, First \\ Affiliated Hospital, Guangzhou University \\ of Chinese Medicine, Guangzhou, \\ 5 10405, People's Republic of China; \\ ${ }^{3}$ Department of Pathology, School of \\ Basic Medical Sciences, Southern Medical \\ University, Guangzhou, 510515, People's \\ Republic of China
}

Correspondence: Hong Shen

Tel +86 20-61648227

$\mathrm{Fax}+8620-61648312$

Email shenhong2010168@163.com
Purpose: Cisplatin is one of the most widely used antineoplastic drugs but has limited therapeutic effects due to nephrotoxicity. The aim of this study was to determine the possible renoprotective effect of the antioxidant raloxifene on cisplatin-induced nephrotoxicity in mice.

Materials and Methods: Cisplatin-induced acute renal injury was established in female C57 mice that were treated with saline (normal control) or raloxifene over a 7-day period. The body weight of the mice was recorded. Histopathological examinations of the kidney tissues were performed using H\&E, PAS staining and TEM. The histomorphology of liver and other organs was observed by H\&E staining. The serum levels of creatinine, blood urea nitrogen (BUN), alanine transaminase (ALT) and glutamic oxalacetic transaminase (AST) were analyzed by specific kits. Superoxide dismutase (SOD) and glutathione (GSH) activity, and the content of malondialdehyde (MDA) in the kidney, liver homogenates and HK-2 cells were measured by WST- 8 and thiobarbituric acid colorimetric methods. Moreover, the mitochondrial structures of HK-2 cells were performed using TEM. The viability and proliferation of HK-2 cells were examined by CCK-8 and EdU incorporation assays. The mitochondrial membrane potential was measured by JC-1 fluorescence.

Results: Raloxifene significantly reduced the levels of serum creatinine, urea, ALT and AST in the cisplatin-treated mice, and alleviated cisplatin-induced renal and hepatic tissue injury. Furthermore, raloxifene also increased the activity of GSH and SOD in the renal tissues and HK-2 cells, and reduced MDA levels, thereby limiting oxidative stress in the kidney.

Conclusion: Raloxifene protected against cisplatin-induced nephrotoxicity by activating the antioxidant system, along with alleviating liver damage. It should be considered as a potential adjuvant in cisplatin-based chemotherapeutic protocols.

Keywords: raloxifene, cisplatin, nephrotoxicity, hepatotoxicity, oxidative stress

\section{Introduction}

Cisplatin, aka cis-diamminedichloroplatinum (CDDP, DDP), is the most commonly used chemotherapeutic against multiple solid tumors despite its considerable side effects. ${ }^{1,2}$ It induces DNA damage in tumor cells by forming adducts with nuclear DNA, which inhibits DNA replication and transcription, eventually leading to apoptosis. ${ }^{3}$ In addition, cisplatin also triggers production of reactive oxygen species (ROS) via mitochondrial DNA (mtDNA) damage, and the resulting oxidative damage to the proteins involved in electron transport chain exacerbates mitochondrial dysfunction. ${ }^{4,5}$ However, almost one-third of the patients receiving the therapeutic dose of cisplatin experience nephrotoxicity. Therefore, it is crucial to explore drugs that reduce cisplatin-induced renal injury and can be administered as an adjuvant with cisplatin. 
Oxidative stress is the primary mechanism underlying cisplatin-induced nephrotoxicity. In addition to increasing ROS production, cisplatin also inhibits the activity of antioxidant enzymes. The accumulation of free radicals in the cisplatin-treated cells leads to the peroxidation of membrane lipids, which alters membrane permeability and impairs cellular function. Raloxifene (Ral) is a second generation Selective Estrogen Receptor Modulator (SERM) approved by the Food and Drug Administration (FDA) for the prevention of breast cancer and postmenopausal women osteoporosis. ${ }^{6}$ A population-based, case-control study provides evidence that raloxifene reduces endometrial cancer risk in women. ${ }^{7}$ Osmanova et al have shown raloxifene is a phenylthiophene compound with antioxidant effects. ${ }^{8}$ Previous studies have shown that raloxifene can reduce oxidative stress and lipid peroxidation in the rat brain, ${ }^{8,9}$ and protects glucosedeficient astrocytes from oxidative injury. ${ }^{10}$ In addition, raloxifene can improve hypertension-induced endothelial dysfunction and vascular injury in rats by increasing endothelial NO synthase activity and reducing estrogen receptor-dependent generation of reactive oxygen species in vascular cells. ${ }^{11}$ Recently, a study shows that raloxifene can postpone adjacent segmental intervertebral disk degeneration development in rats through inhibiting extracellular matrix metabolic imbalance, nucleus pulposus cell apoptosis, and vertebral osteoporosis. ${ }^{12}$ In addition, raloxifene may inhibit molecules involved in the inflammatory processes associated with COVID-19. ${ }^{13,14}$ However, whether raloxifene could reduce cisplatin-induced renal injury is unclear. The aim of this study was to analyze the potential reno-protective effects of raloxifene in a mouse model of cisplatin-induced acute renal injury, in order to provide an experimental basis for using raloxifene as an adjuvant in cisplatin-based chemotherapy.

\section{Materials and Methods} Drugs and Chemicals

Cisplatin (molecular formula: Cl2H6N2Pt, molecular weight: 300.05) was purchased from Jiangsu Hansoh Pharmaceutical Group Co. Ltd., and raloxifene (molecular formula: C28H27NO4S.HCl, molecular weight: 510.05) from Dalian Meilun Biotechnology Co. Ltd. RIPA (radio immunoprecipitation assay) buffer, phenylmethanesulfonyl fluoride (PMSF), BCA protein quantification kit, and the superoxide dismutase (SOD), malondialdehyde (MDA), glutathione (GSH) detection kits, JC-1 mitochondrial membrane potential assay kit and 5-Ethynyl-2'deoxyuridine (EdU) Cell Proliferation Kit with Alexa Fluor 594 were purchased from Beyotime Biotechnology. Hematoxylin and eosin (H\&E) staining solutions, cell counting kit 8 (CCK-8) were purchased from KeyGEN Biotechnology Co. Ltd. Periodic acid-Schiff (PAS) staining solutions and corn oil were purchased from Solaibao Technology Co. Ltd., ethanol and xylene from Aladdin reagents, and the creatinine and urea nitrogen detection kits from Kehua Biological Engineering Co. Ltd. Primary antibody: anti-Bcl-2, anti-Bax, anti- $\beta$-actin were purchased from Wanleibio Company (Shenyang, China). HRP-labeled secondary antibody was purchased from Proteintech Company (Wuhan, China).

\section{Cisplatin-Induced Acute Renal Injury Model and Treatment Regimen}

Female C57 mice (SPF grade, weighing 18-22 g) were purchased from Hunan Slyke Experimental Animal Co. Ltd. (animal license number SCXK (Xiang)-2019-0004). All experimental procedures were performed in accordance with the Guide for the Care and Use of Laboratory Animals (National Research Council Publication, 8th Edition, 2011) and were in compliance with the Chinese Regulations for the Administration of Affairs Concerning Experimental Animals. The animal experimental protocol was approved by the Animal Experimental Ethics Committee of South China University of Technology (No. 2018051). The mice were raised in independent ventilation box cages at $23-25^{\circ} \mathrm{C}, 55-65 \%$ humidity and 12 $\mathrm{h}$ circadian rhythm with ad libitum access to food and water. Thirty mice were randomly divided into the following groups and subjected to the respective regimens for 7 days: (1) blank control - intragastric gavage of normal saline, (2) raloxifene $-10 \mathrm{mg} / \mathrm{kg}$ raloxifene via intragastric gavage, (3) Cisplatin - daily saline gavage from day 1 to day 5 , and $25 \mathrm{mg} / \mathrm{kg}$ cisplatin injected intraperitoneally $2 \mathrm{~h}$ after saline administration on the fifth day, (4) combination $1-10 \mathrm{mg} / \mathrm{kg}$ raloxifene from day 1 to day 5 , and cisplatin $(25 \mathrm{mg} / \mathrm{kg})$ injection $2 \mathrm{~h}$ after raloxifene on the fifth day, and (5) combination 2 - identical as above with $12.5 \mathrm{mg} / \mathrm{kg}$ cisplatin (half dose).

The mice were weighed every day during the treatment course prior to drug administration. The mice were euthanized on day 7, and the liver and kidneys were removed. The kidney index was calculated as (kidney mass/body mass) $\times 100 \%$ and the liver index as (liver mass/body 
mass) $\times 100 \%$ were calculated. The major organs (liver, kidneys, spleen, lungs, heart and intestines) were partly snap frozen in liquid nitrogen and the remaining were fixed in $4 \%$ paraformaldehyde for $48 \mathrm{~h}$. The eyeballs were removed and bled, and the blood was allowed to coagulate for $30 \mathrm{~min}$. The supernatant was centrifuged at $3500 \mathrm{~g}$ and the serum was collected for biochemical analysis.

\section{Hematoxylin-Eosin (H\&E) Staining}

The fixed tissues were washed, dehydrated across an ethanol gradient, cleared in xylene, and embedded in paraffin. The blocks were sliced into $4 \mu \mathrm{m}$-thick sections, dewaxed and hydrated. The sections were stained with hematoxylin, cleared with $1 \%$ hydrochloric acid alcohol, and counterstained with eosin. After dehydration and dewaxing, the stained sections were sealed with neutral gum and observed under a high-power microscope.

\section{Periodic Acid-Schiff (PAS) Staining}

Periodic acid-Schiff (PAS) stain kits were purchased from Solaibao Technology Co. Ltd. (Beijing, China). Briefly, paraffin-embedded sections were dewaxed in xylene, rehydrated in ethanol, and rinsed in distilled water. After oxidation in periodic acid for 15 minutes, each section was stained with Schiff reagent for 10 minutes, rinsed with distilled water for 5 minutes, and counterstained with hematoxylin for 2 minutes. The sections were subsequently washed, dehydrated, and sealed with a neutral resin for microscopic examination.

\section{Biochemical Assays}

Serum creatinine (SCr), blood urea nitrogen (BUN), alanine transaminase (ALT), and glutamic oxalacetic transaminase (AST) levels were detected by automatic biochemical analyzer according to the manufacturer's instructions. The liver and kidney tissues were homogenized on an ice bath, and the cultured HK-2 cells were harvested and washed with PBS. The tissue homogenates and cell suspensions were resuspended in the suitable buffers provided with the BCA protein detection kit, and specific MDA, SOD and GSH kits (Beyotime Biotechnology, China), and the assays were performed according to the manufacturer's instructions. SOD activity was measured in terms of the absorbance at $450 \mathrm{~nm}$. The GSH content was measured via DTNB reaction in the presence of NADPH, and the absorbance at $412 \mathrm{~nm}$ was recorded. MDA absorbance was measured at $530 \mathrm{~nm}$. Each experiment was performed in triplicates.

\section{Transmission Electron Microscope (TEM) Analysis}

Freshly prepared kidney tissues of mice and HK-2 cells treated with drugs were immediately fixed for 2 hours in $2.5 \%$ glutaraldehyde in $0.1 \mathrm{M}$ phosphate-buffered saline $(\mathrm{pH}=7.4)$ and post-fixed in $1 \%$ OsO4, followed by washing in distilled water and en bloc staining in $3 \%$ uranyl acetate. Dehydration was conducted using a 70$100 \%$ graded ethanol. The tissues and HK-2 cells were then embedded, respectively, in Epon polymer and ultrathin sections $(70 \mathrm{~nm})$ were obtained with a diamond knife on a Reichert Jung ultramicrotome (Leica, Germany), stained with uranyl acetate-lead citrate and observed with a JEM-1400plus transmission electron microscope (Japan Electron Optics Laboratory Co., Ltd., Japan). The TEM images with magnifications ranging from 5000× were analyzed for the examination of the morphology of subcellular constituents and structures, 20,000×were analyzed for the examination of the mitochondrial structure of HK-2 cells.

\section{Cell Culture}

The human renal tubular epithelial cell-line HK-2 was a kind gift from Professor Ji-Yan Chen of Guangdong Provincial people's Hospital affiliated to South China University of Technology. The use of the cell line was approved by the institutional Research Ethics Committee of Guangdong Provincial People's Hospital (No:KYZ-2021-2151-01). The HK-2 cells were cultured in DMEM/F12 medium containing 15\% fetal bovine serum and $1 \%$ penicillin and streptomycin at $37^{\circ} \mathrm{C}$ under $5 \%$ $\mathrm{CO} 2$. The medium was changed every 2 days, and cells in the logarithmic growth phase were used for all experiments.

\section{CCK-8 Assay}

HK-2 cells were seeded in a 96-well plate at the density of 1000 cells/well, and allowed to adhere for $24 \mathrm{~h}$. Following $24 \mathrm{~h}$ treatment with different concentrations of raloxifene (0.01-10 $\mu \mathrm{M})$, cisplatin $(0.5-2 \mu \mathrm{g} / \mathrm{mL})$, or the combination of cisplatin $(2 \mu \mathrm{g} / \mathrm{mL})$ and raloxifene $(1 \mu \mathrm{M}$ or $10 \mu \mathrm{M})$, the cells were washed twice with complete medium, and incubated with $10 \mu \mathrm{L}$ CCK8 reagent (Beyotime Biotechnology, Beijing) in $100 \mu \mathrm{L}$ medium for $2 \mathrm{~h}$. The absorbance at 450 
nm was measured by Microplate Reader (Bio-Tek, Winooski, VT, USA), and the cell viability was calculated. Results were expressed by the cell viability of results from replicated wells. Cell viability was calculated as follows: (sample group-blank group)/(untreated group-blank group) $* 100 \%$, where the blank group represents the absorbance value of PBS (phosphate buffered saline) in the absence of cells.

\section{EdU Assay}

The EdU assay was performed to determine cell proliferation. HK-2 cells were seeded onto cover slips in 12-well plate at the density of 50,000 cells/well, and treated with cisplatin $(2 \mu \mathrm{g} / \mathrm{mL})$ in the presence or absence of raloxifene $(1 \mu \mathrm{M}$ or $10 \mu \mathrm{M})$ for $24 \mathrm{~h}$. After rinsing in PBS, the cell line was incubated with $1 \mu \mathrm{M} E d U$ at $37^{\circ} \mathrm{C}$ for $2 \mathrm{~h}$. Following incubation, the cell line was fixed with $4 \%$ paraformaldehyde for $15 \mathrm{~min}$, incubated with $0.5 \%$ Triton X-100 for $15 \mathrm{~min}$, and washed 3 times with PBS. Subsequently, the cell line was incubated with a $0.5 \mathrm{~mL}$ click additive solution at ambient temperature for 30 min in the dark. After counterstaining with DAPI at $37^{\circ} \mathrm{C}$ for $10 \mathrm{~min}$, the cells were observed under a fluorescence microscope (Zeiss, Ax10 Axio, Germany).

\section{H\&E Staining of Cell Climbing Slices}

HK-2 cells were seeded onto cover slips in 12-well plate at the density of 50,000 cells/well, and treated with cisplatin $(2 \mu \mathrm{g} / \mathrm{mL})$ in the presence or absence of raloxifene $(1 \mu \mathrm{M}$ or $10 \mu \mathrm{M}$ ) for $24 \mathrm{~h}$. The cell climbing tablets were then stained with H\&E as described above, and observed under a light microscope (Olympus, Tokyo, Japan).

\section{Mitochondrial Membrane Potential Detection}

HK-2 cells were seeded onto cover slips (NEST Biotechnology, China) in a 12-well plate (NEST Biotechnology, China) at the density of 50,000 cells/well, and treated with cisplatin with or without raloxifene as described for $24 \mathrm{~h}$. The cells were fixed with $4 \%$ paraformaldehyde and stained with $5 \mathrm{mg} / \mathrm{L} \mathrm{JC}-1$ (Mitochondrial Membrane Potential Detection Kit) (Beyotime Biotechnology, China) at $37^{\circ} \mathrm{C}$ for $1 \mathrm{~h}$. After counterstaining with DAPI at $37^{\circ} \mathrm{C}$ for $10 \mathrm{~min}$, the cells were observed under a fluorescence microscope (Zeiss, Ax10 Axio, Germany). JC-1 accumulates in the mitochondrial matrix in a red-fluorescing polymeric form at high membrane potential and as a monomer emitting green fluorescence signals at low mitochondrial membrane potential. The ratio of green/red JC-1 fluorescence was evaluated to assess membrane depolarization.

\section{Western Blot Assay}

HK-2 cells were lysed with RIPA lysis buffer on the ice box. Protein concentrations were detected with BCA protein assay kit (Beyotime Biotechnology, Shanghai, China). Proteins were separated by electrophoresis on $10 \%$ SDSpolyacrylamide gel, transferred on the polyvinylidene difluoride (PVDF) membrane (Millipore, Burlington, MA, USA), and blocked by $5 \%$ fat-free milk at room temperature for 2 hours. The membranes were covered with primary antibodies and incubated at $4{ }^{\circ} \mathrm{C}$ overnight, Bcl-2, Bax, $\beta$-actin at 1:1000 (Wanleibio, Shenyang, China). Then, membranes were incubated with HRPlabeled secondary antibody (1:20,000) (Proteintech, Wuhan, China) at room temperature for $1 \mathrm{~h}$ after being washed by TBST for three times. The interested proteins were detected by ECL chemiluminescence. The data of blot bands were analyzed with Image J software.

\section{Statistical Analysis}

SPSS 26.0 software was used for statistical analysis. The data are expressed as mean \pm standard deviation of at least three independent experiments. One-way ANOVA and the post-hoc SNK-q test were used to compare different groups. $\mathrm{P}<0.05$ was considered statistically significant. The bar graphs present the mean \pm standard deviation (SD) of separate experiments. * indicates $\mathrm{p}<0.05$, and ** indicates $\mathrm{p}<0.01, * * *$ indicates $\mathrm{p}<0.001$, which were all considered significance. Each assay was performed independently three times. All graphs were made using GraphPad Prism 8.0.

\section{Results}

\section{Raloxifene Attenuates Cisplatin-Induced Renal and Hepatic Injury}

The reno-protective effect of raloxifene was evaluated in terms of the renal/body mass index, and the BUN and serum creatinine ( $\mathrm{SCr}$ ) levels. As shown in Figure 1A, the cisplatin-treated mice showed significant decline in body weight compared to the normal control group. However, pre-treatment with raloxifene increased the body weight compared to cisplatin control $(\mathrm{P}<0.05)$. Furthermore, cisplatin-treated group showed significant 

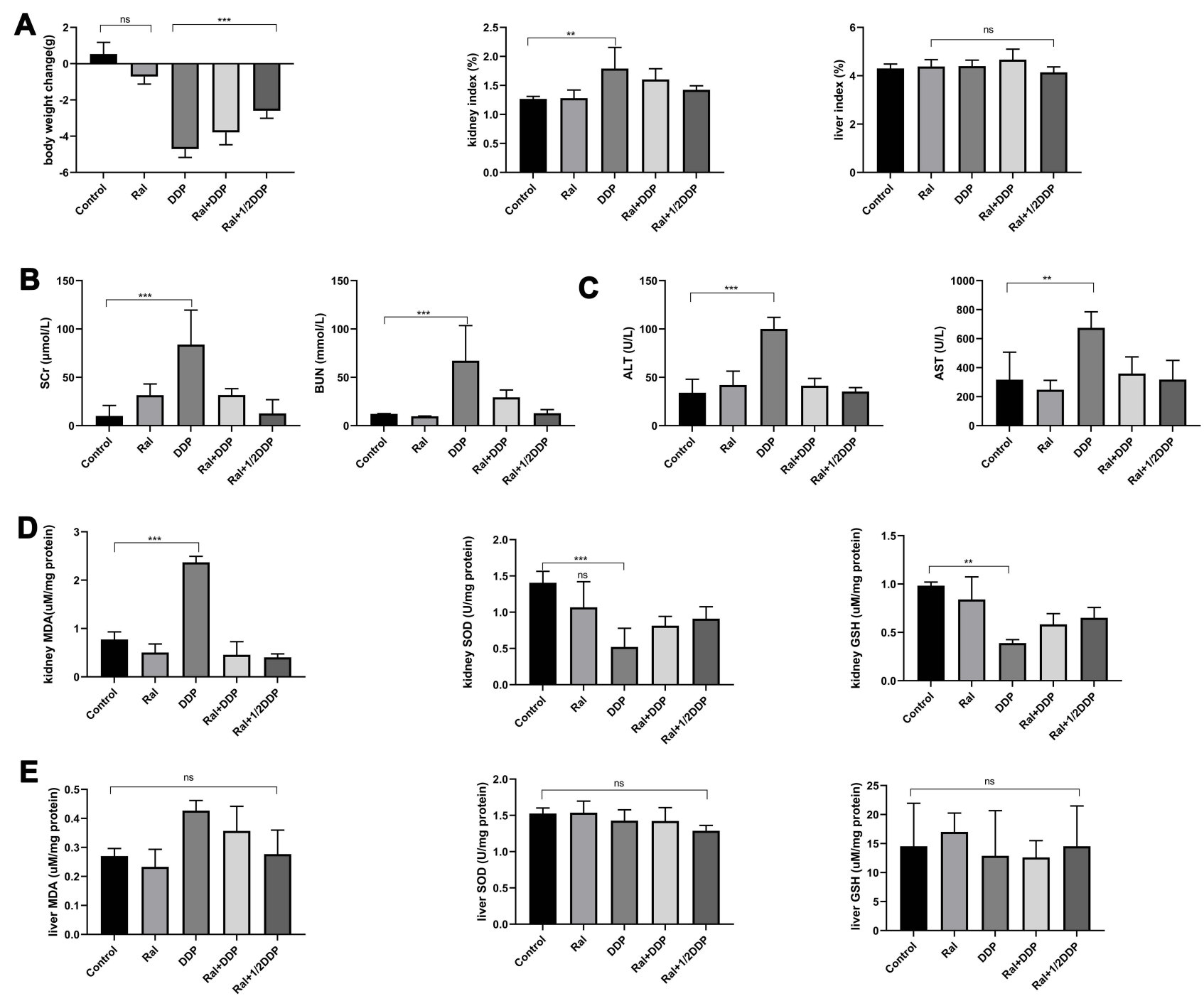

Figure I Raloxifene attenuates cisplatin-induced renal injury and liver injury in female C57 mice. Effect of raloxifene and cisplatin on the body weight, kidney/body weight index and liver/body weight index (A), and the levels of serum creatinine, BUN (B), ALT and AST (C). (D) MDA, SOD and GSH levels in the kidney tissues of mice treated with Ral (10 mg/kg), DDP (25 mg/kg), Ral (10 mg/kg) + DDP $(25 \mathrm{mg} / \mathrm{kg})$ and Ral (10 mg/kg) + I/2 DDP (I2.5 mg/kg). Ral - raloxifene; DDP - cisplatin. Values are the mean \pm SD $(\mathrm{n}=6), * * \mathrm{P}<0.01$, ***P $<0.001$ vs Control.

gain in renal/body mass index when compared with control $(\mathrm{P}<0.01)$, which was abrogated by raloxifene pretreatment $(\mathrm{P}<0.05)$.

As shown in Figure 1B, the contents of serum creatinine and urea nitrogen in the model group were significantly higher than that in the normal group $(\mathrm{P}<0.05)$, indicating glomerular filtration dysfunction and impaired renal function. Compared with the model group, the combined administration of raloxifene could significantly reduce the contents of serum creatinine and urea nitrogen $(\mathrm{P}<0.05)$, but did not return to the normal level.

To confirm the renoprotective effect of raloxifene in cisplatin-treated mice, the morphological changes in the kidneys tissues were assessed using H\&E, PAS staining and TEM. As illustrated in Figure 2, kidneys from mice of either the vehicle control or raloxifene alone group displayed normal renal architecture. DDP alone treatment resulted in severe renal tubular damage, characterized by severe degeneration, necrosis and desquamation of renal tubular epithelial cells, dilatation of the tubular lumens and cast formation in the lumens of tubules, dilation of Bowman's capsular space. TEM images show dramatic mitochondrial swelling with a loss of cristae in epithelial cells (Figure 2). However, pretreatment with raloxifene at $10 \mathrm{mg} / \mathrm{kg}$ significantly attenuated the pathological changes in cisplatin-treated mice. Although some mitochondria are rounded in DDP plus Ral, there is much less matrix swelling and cristae membranes are preserved. Importantly, treatment with $10 \mathrm{mg} / \mathrm{kg}$ raloxifene 


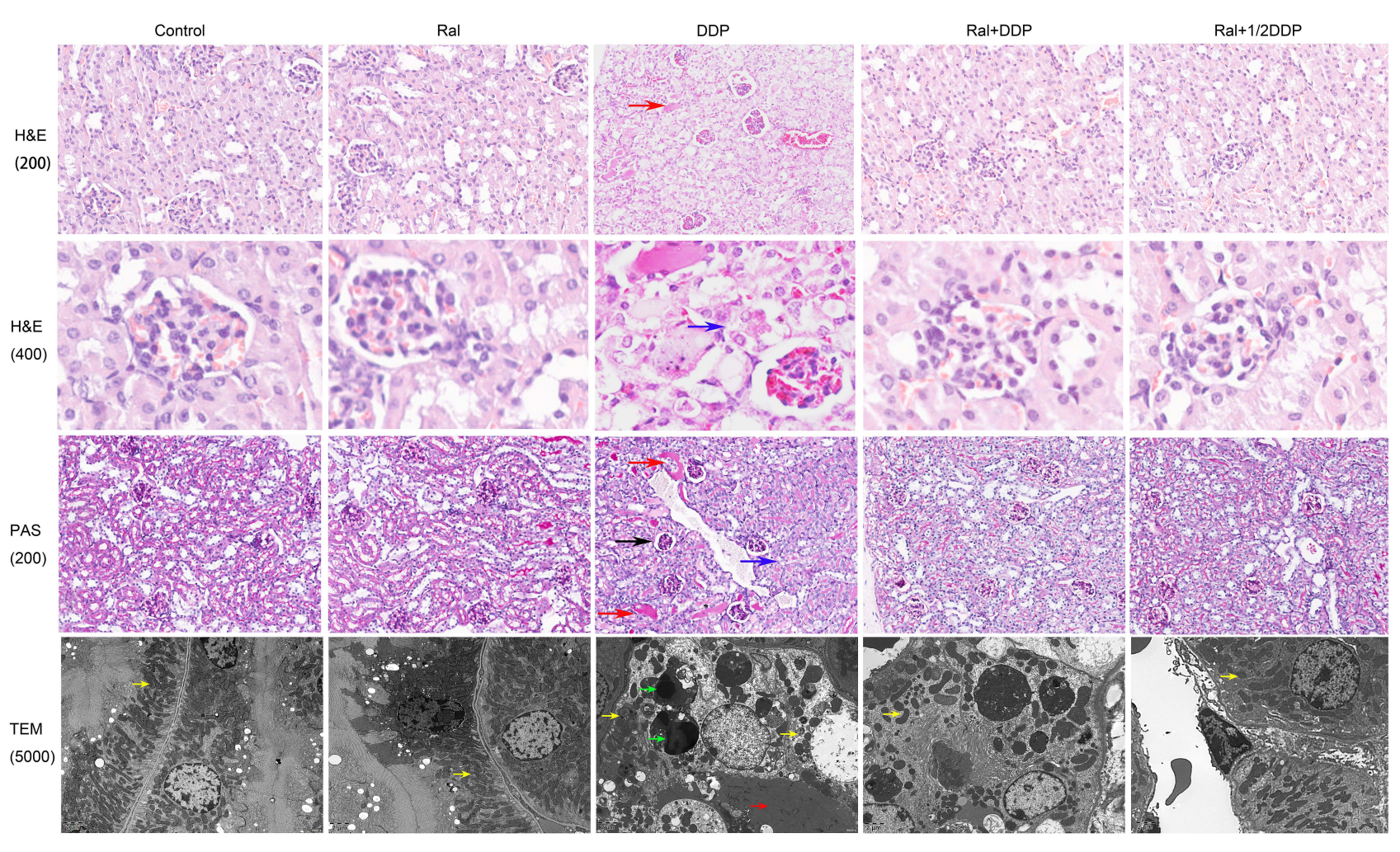

Figure 2 Raloxifene attenuates cisplatin-induced renal injury in mice. Histopathological examination of the kidney tissues of Control, Ral ( 0 mg/kg), DDP (25 mg/kg), Ral $(10 \mathrm{mg} / \mathrm{kg})+$ DDP $(25 \mathrm{mg} / \mathrm{kg})$ and Ral $(10 \mathrm{mg} / \mathrm{kg})+1 / 2$ DDP $(12.5 \mathrm{mg} / \mathrm{kg})$ groups. H\&E-stained and PAS-stained sections were observed under a light microscope (Obj 200 $\times, 400 \times, 200 \times)$. More severe tubular injury was observed in DDP group mice compared with other mice. Representative H\&E-stained and PAS-stained sections indicate acute kidney injury in DDP group, blue arrows denote severe degeneration, necrosis and desquamation of renal tubular epithelial cells, red arrows indicate dilatation of the tubular lumens and cast formation in the lumens of tubules, black arrow indicates dilation of Bowman's capsular space. TEM images show dramatic mitochondrial swelling with a loss of cristae in epithelial cells in DDP group. Although most mitochondria are rounded in Ral+(I/2)DDP and Ral+DDP group, there is much less matrix swelling and cristae membranes are preserved, yellow arrows denote mitochondria, red arrow indicate cast formation in the lumens of tubules, green arrows indicate autolysosomes in the epithelia of injured kidneys.

alone caused no alterations in renal function and morphology. The results consistent with the biochemical indices.

Although DDP did not increase the liver index significantly $(\mathrm{P}>0.05$; Figure $1 \mathrm{~A})$, the serum levels of glutamic pyruvic transaminase and glutamic oxaloacetic transaminase were markedly higher in the model group compared to the control $(\mathrm{P}<0.05$; Figure 1C). In addition, DDP treatment induced liver tissue damage, including hydro-degeneration and fatty degeneration of hepatocytes (Figure 3). Raloxifene significantly improved the serological and histopathological

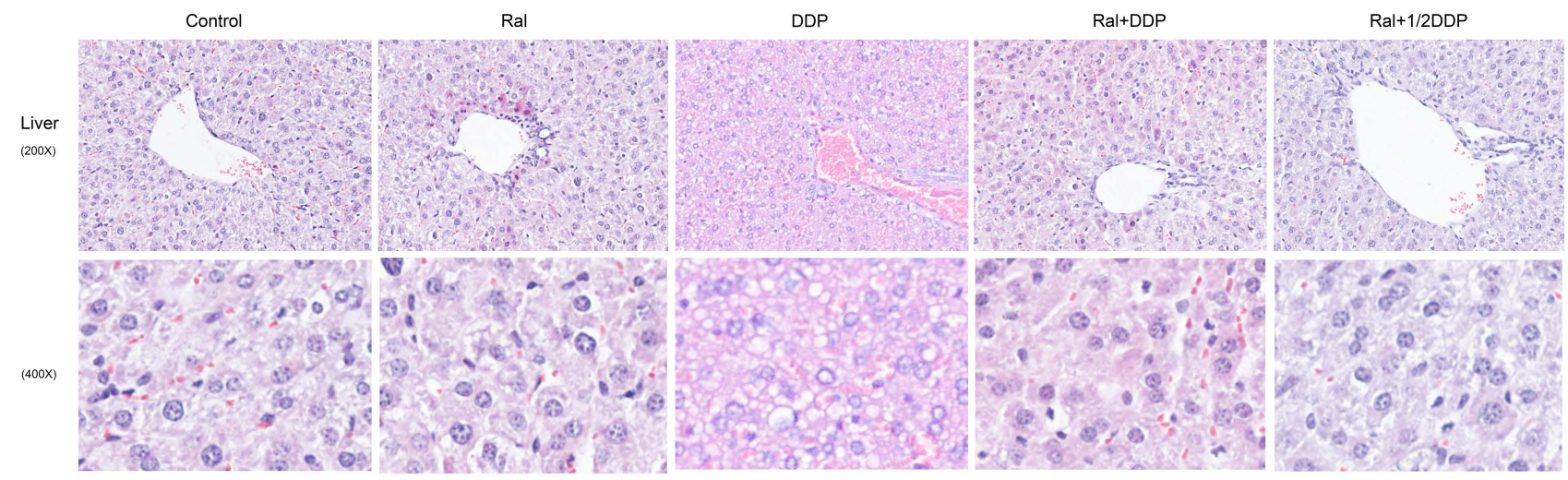

Figure 3 Effect of DDP and Ral on hepatic pathological changes. Liver histology in mice treated with DDP or DDP plus Ral. The major histopathological change induced by DDP in mice liver was hydro-degeneration and microvesicular steatosis. Ral treatment suppressed the DDP-induced alterations, with diminished fatty infiltration and minimal steatosis. H\&E-stained sections were observed under a light microscope $(200 \times$ and $400 \times)$. 
indices of liver function $(\mathrm{P}<0.05)$. The minimal effect of cisplatin on the liver index can be attributed to the short modelling duration. In addition, the liver is not the main organ of cisplatin metabolism, and has a robust compensatory function. Therefore, the serological changes occurred prior to tissue damage. The lung, heart, intestine, and spleen did not exhibit any significant histological change, which can be attributed to the short modeling period and the fact that these organs are not the main metabolic targets of cisplatin (Figure 4).

\section{Raloxifene Mitigates Oxidative Stress and Apoptosis in Cisplatin-Treated Renal Cells}

As shown in Figure 1D, cisplatin significantly reduced the glutathione content and SOD activity in the kidneys, and increased MDA levels compared to the control $(\mathrm{P}<0.05)$, indicating oxidative stress. Raloxifene significantly increased the content of GSH and SOD activity, and decreased the level of MDA $(\mathrm{P}<0.05)$. However, glutathione, MDA content and SOD activity in the liver were similar across the five groups (Figure 1E), indicating that cisplatin-induced liver injury, as well as the protective effects of raloxifene, involves other mechanisms.

The toxicity of cisplatin and raloxifene on HK-2 cells was tested at different concentrations over a time frame of $24 \mathrm{~h}$ via CCK-8 assay. Cisplatin had the potent growth-inhibitory effect on HK-2 cells ( $\mathrm{IC} 50=2 \mu \mathrm{g} / \mathrm{mL}$ ), while raloxifene alleviated the inhibitory effects of HK-2 cells in a dose-dependent manner (Figure 5A). Raloxifene attenuates cisplatin-induced HK-2 cells apoptosis. As shown in Figure 5C, cisplatin caused obvious increase of the proapoptotic protein Bax and decrease of the antiapoptotic protein Bcl-2. Inhibition of Bax activity and upregulation of Bcl-2 were evidenced by combine with raloxifene at $1 \mu \mathrm{M}$ or $10 \mu \mathrm{M}$. To further confirm raloxifene alleviated cisplatin-induced the inhibitory effects of HK-2 cell proliferation, EdU incorporation was used, and the results are shown in Figure 6A. It was observed that DDP induction could remarkably suppressed the proliferation of HK-2 cells compared with the control groups, while DDP plus Ral treatment alleviated the inhibitory effects of HK-2 cells proliferation compared with DDP group.

In addition, cisplatin-induced shrinkage, cell roundness and cytoplasmic vacuolization were also rescued by raloxifene (Figure 5D). TEM analysis shows mitochondrial

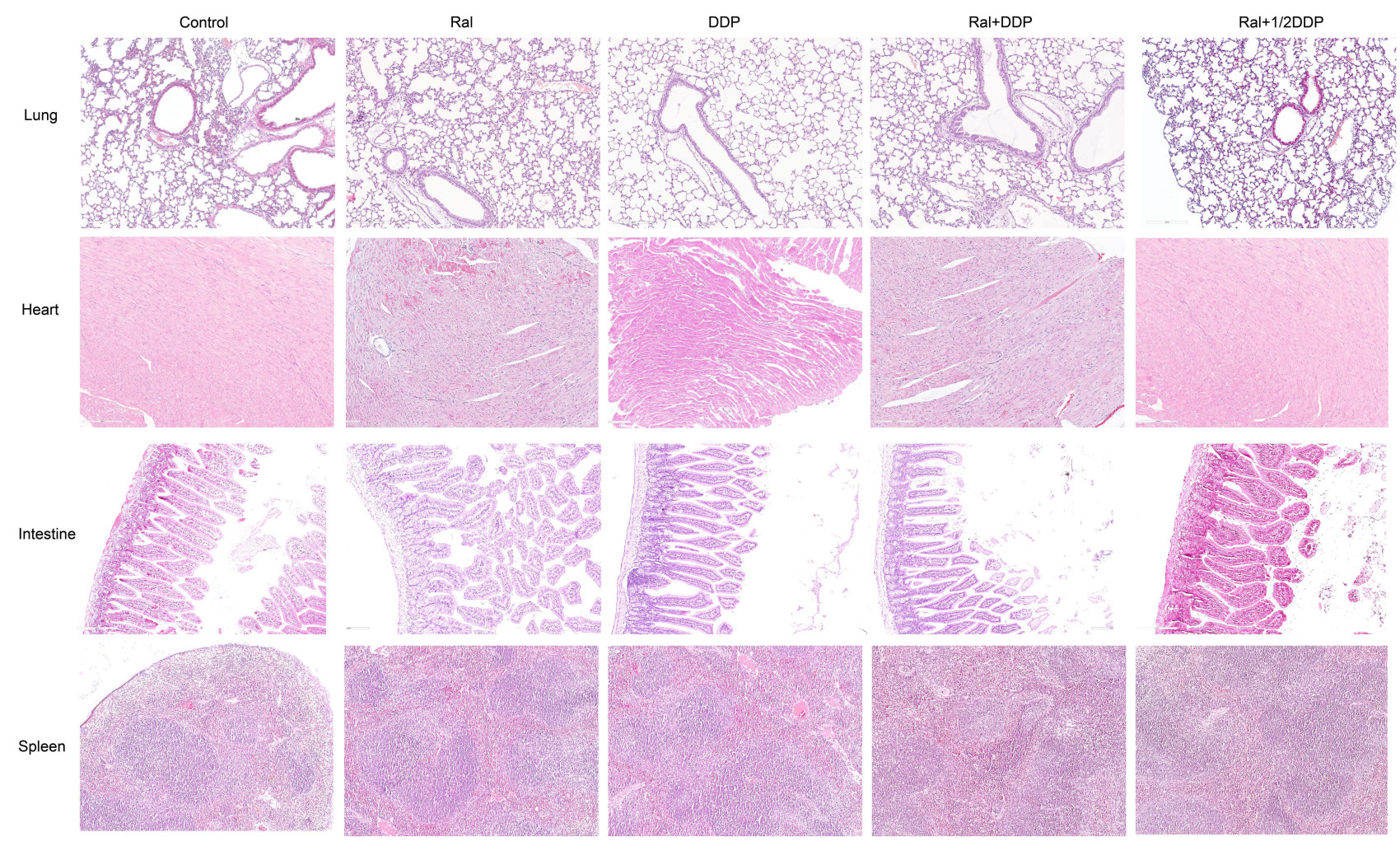

Figure 4 Histopathological examination of the lungs, hearts, intestines and spleens of Control, Ral (I0 mg/kg), DDP (25 mg/kg), Ral (I0 mg/kg) + DDP (25 mg/kg) and Ral $(10 \mathrm{mg} / \mathrm{kg})+1 / 2$ DDP $(12.5 \mathrm{mg} / \mathrm{kg})$ groups. H\&E-stained sections were observed under a light microscope $(100 \times)$. 

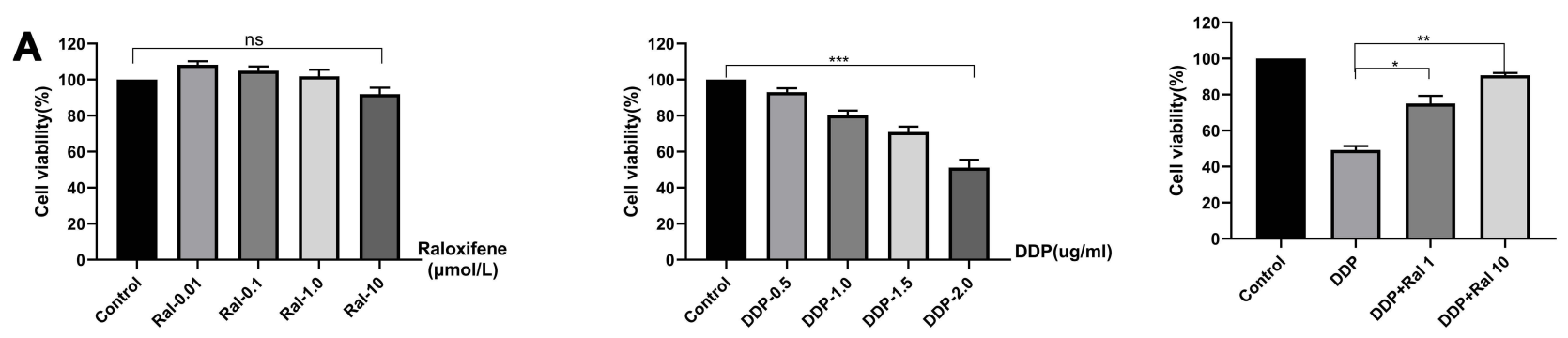

B

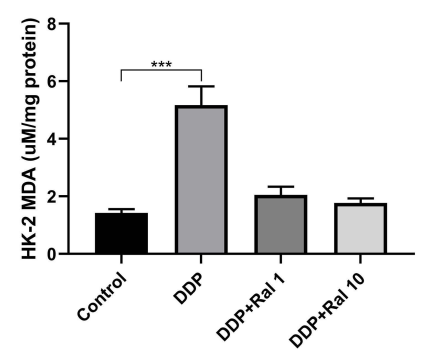

C

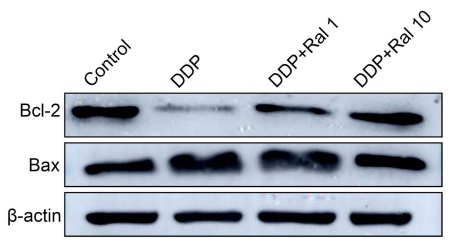

Control
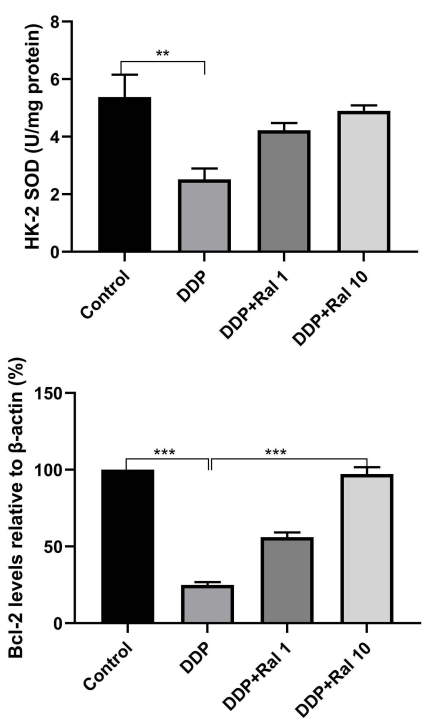
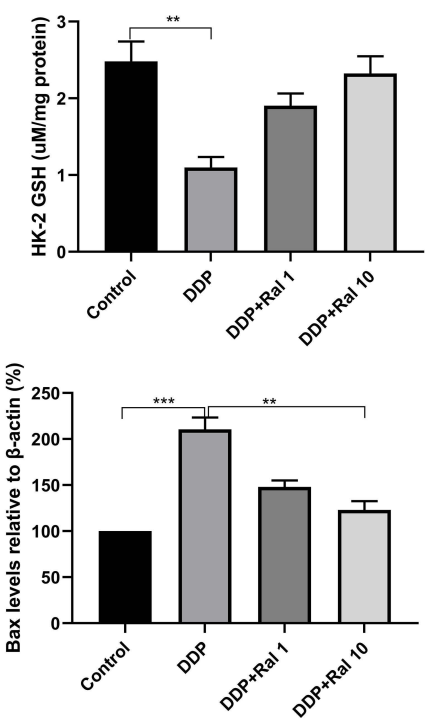

DDP+Ral 10
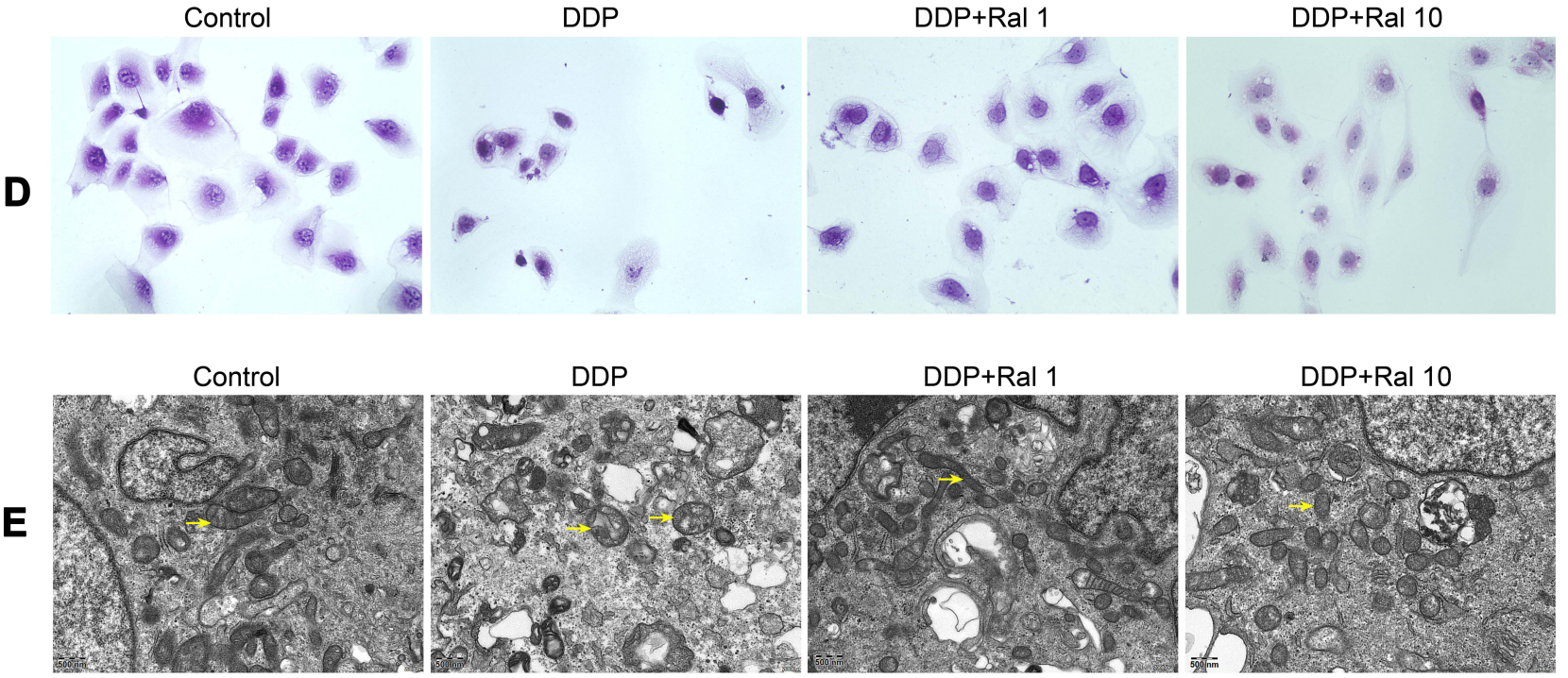

Figure 5 Raloxifene attenuated cisplatin-induced oxidative stress in HK-2 cells. (A) Effect of raloxifene on cisplatin-induced cytotoxicity in HK-2 cells. HK-2 cells were treated with indicated concentrations of raloxifene $(0.0 \mathrm{l}-10 \mu \mathrm{M})$, cisplatin $(0.5-2 \mu \mathrm{g} / \mathrm{mL})$, cisplatin $(2 \mu \mathrm{g} / \mathrm{mL})+$ raloxifene $(\mathrm{I} \mu \mathrm{M}$ or $\mathrm{I0} \mu \mathrm{M})$ for $24 \mathrm{~h}$. (B) $\mathrm{MDA}$, SOD and GSH levels in HK-2 cells. HK-2 cells were treated with indicated concentrations of cisplatin $(2 \mu \mathrm{g} / \mathrm{mL})$, cisplatin $(2 \mu \mathrm{g} / \mathrm{mL})+\mathrm{raloxifene}(\mathrm{I} \mu \mathrm{M}$ or $\mathrm{I0} \mu \mathrm{M})$ for $24 \mathrm{~h}$. $(\mathbf{C})$ Western blot assay for the detection of Bcl-2 and Bax expression in each group and quantifications: $\beta$-actin was used to normalize protein expression levels. (D) H\&E morphological staining of HK-2 cells (original magnification: $\times 200$ ). Values are the mean $\pm \mathrm{SD}$ of three independent experiments. $* \mathrm{P}<0.05$, $* * \mathrm{P}<0.0 \mathrm{I}$, $* * * \mathrm{P}<0.00 \mathrm{I}$ vs control. (E) TEM analysis shows mitochondrial swelling and severe disruption of mitochondrial structures in DDP-treated HK-2 cells, only a few mitochondria are present and they appear swollen and have lost cristae. TEM picture of control group HK-2 cells, showing elongated mitochondria with normal cristae structure. TEM picture of DDP + Ral $(1 \mu \mathrm{M}$ or $10 \mu \mathrm{M})$ groups HK-2 cells, showing many normal mitochondria are still present, but disrupted mitochondria that are swollen are also observed in a few mitochondria. 

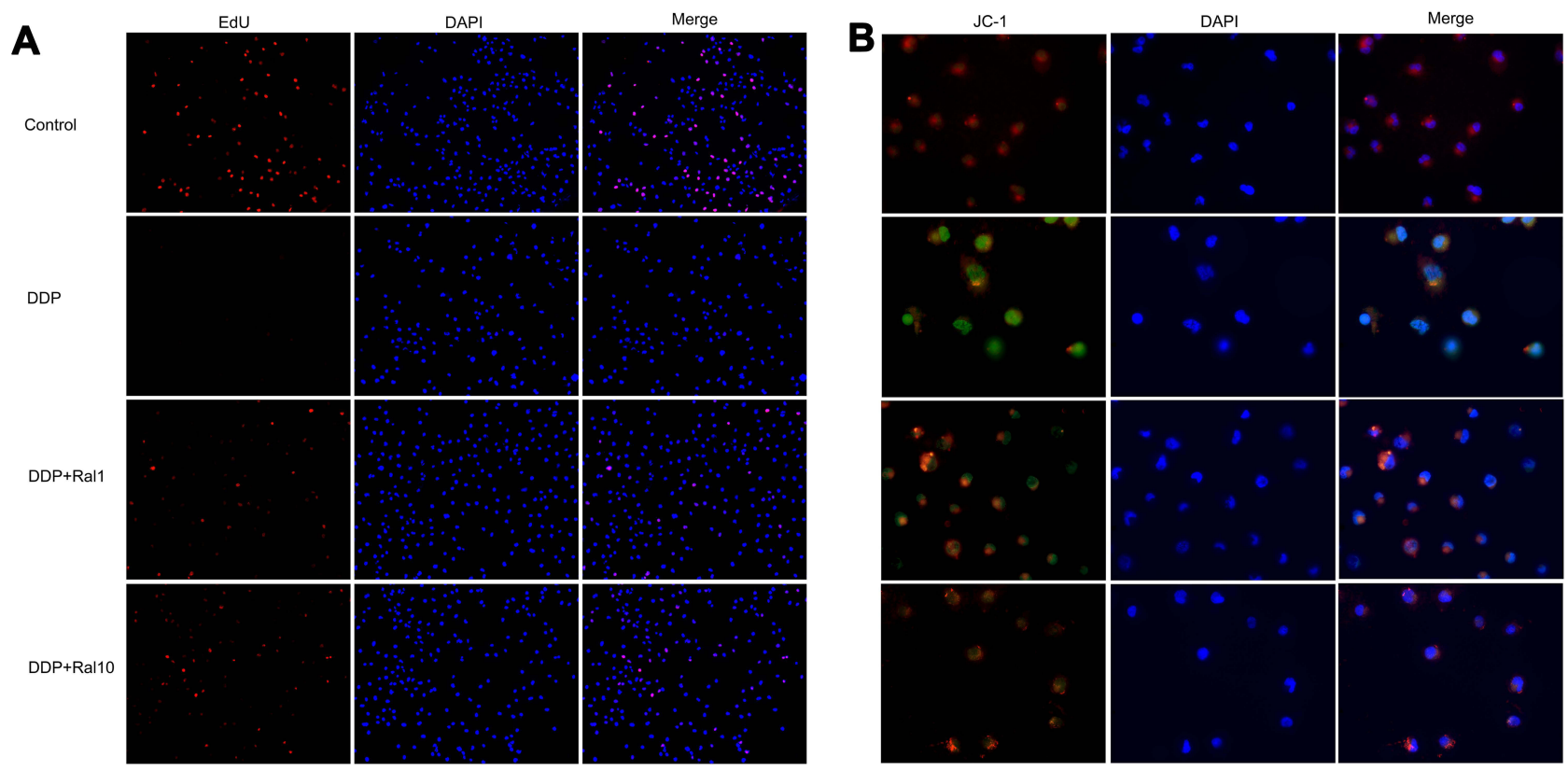

Figure 6 Raloxifene alleviated cisplatin-induced the inhibitory effects of HK-2 cell proliferation and mitochondrial dysfunction. HK-2 cells were treated with the indicated concentrations of cisplatin $(2 \mu \mathrm{g} / \mathrm{mL})$, cisplatin $(2 \mu \mathrm{g} / \mathrm{mL})+$ raloxifene $(I \mu \mathrm{M}$ or $10 \mu \mathrm{M})$ for $24 \mathrm{~h}$. (A) The cell proliferation was measured by EdU fluorescence. The cells were fixed and stained with EdU and DAPI, and observed under a fluorescence microscope (original magnification: $\times 100)$. (B) The mitochondrial membrane potential was measured by JC-I fluorescence. The cells were fixed and stained with JC-I and DAPI, and observed under a fluorescence microscope (original magnification: $\times 400$ ).

swelling and severe disruption of mitochondrial structures in DDP-treated HK-2 cells, only a few mitochondria are present and they appear swollen and have lost cristae. TEM picture of control group HK-2 cells, showing elongated mitochondria with normal cristae structure. TEM picture of DDP+Ral $(1 \mu \mathrm{M}$ or $10 \mu \mathrm{M})$ groups HK-2 cells, showing many normal mitochondria are still present, but disrupted mitochondria that are swollen are also observed in a few mitochondria (Figure 5E). Consistent with the in vivo results, cisplatin enhanced the oxidative stress in HK-2 cells, and raloxifene normalized the indices of glutathione, MDA and SOD activity (Figure 5B). In addition, cisplatin-treated cells showed significant mitochondrial membrane depolarization, as indicated by the high green/red JC-1 fluorescence intensity ratio, compared to cells pre-treated with raloxifene $(1 \mu \mathrm{M}$ or $10 \mu \mathrm{M})$. Thus, raloxifene also alleviated cisplatin-induced mitochondrial dysfunction in the renal cells (Figure 6B).

\section{Discussion}

We demonstrated the protective effects of raloxifene against cisplatin-induced nephrotoxicity and hepatotoxicity a mouse model, and elucidated the underlying mechanisms. Cancer is a major public health issue associated with high mortality rates. ${ }^{15}$ Cisplatin, the most common first-line chemotherapy drug for most solid tumors, is associated with acute ototoxicity and nephrotoxicity, among other severe side effects. ${ }^{16}$ Cisplatin-based or compatible drugs account for $70-80 \%$ of all anti-tumor chemotherapy regimens in China,${ }^{17}$ and one-third of the patients treated with cisplatin experience kidney damage. ${ }^{18}$ Acute nephrotoxicity associated with high-dose cisplatin can lead to impaired renal function, delayed treatment and other complications. ${ }^{19}$ In addition, acute renal damage also delays cisplatin clearance, ${ }^{20}$ which exacerbates the risk of adverse events. Therefore, it is vital to incorporate an adjuvant in cisplatin regimens that can mitigate its toxic effects. Raloxifene is a phenylthiophene selective estrogen receptor inhibitor with antioxidant activity. ${ }^{8}$ Previous studies have shown that raloxifene can reduce oxidative stress and lipid peroxidation in the rat brain, ${ }^{8,9}$ and protects glucose-deficient astrocytes from oxidative injury. ${ }^{10}$ In addition, raloxifene can improve hypertension-induced endothelial dysfunction and vascular injury in rats by increasing endothelial NO synthase activity and reducing estrogen receptor-dependent generation of reactive oxygen species in vascular cells. ${ }^{11}$ In this study, raloxifene administered at a dose of $10 \mathrm{mg} / \mathrm{kg}$ significantly restored DDP-induced alterations in biochemical markers and antioxidant status in the liver and kidneys. The beneficial role of raloxifene against DDP-induced oxidative damage observed in the present study may be mediated by the inhibition of MDA, increase in GSH and SOD expression. 
Cisplatin decreases the glomerular filtration rate, which reduces blood flow through the renal vessels and may lead to renal ischemia, ${ }^{21}$ loss of renal function and eventually renal failure. In this study, the serum creatinine and urea levels increased significantly in the cisplatin-treated mice, which confirmed the nephrotoxicity of cisplatin as reported by previous studies on rat models. ${ }^{22-24}$ In addition, cisplatin also led to severe histopathological changes in the kidneys, including vacuolization of renal tubular epithelial cells, inter-tubular capillary hyperemia, glomerular mesangial cell proliferation, inflammatory cell infiltration, glomerular atrophy and focal renal hemorrhage. Furthermore, in this study, cisplatin induced liver damage, as indicated by the high AST and ALT levels, as well as hydro-degeneration and fatty degeneration of liver cells in the treated mice, which was consistent with the results of previous studies. ${ }^{25,26}$ Raloxifene significantly improved the serological and histopathological indices of liver function.

Cisplatin triggers oxidative stress in tumor cells by promoting ROS generation, ${ }^{27,28}$ inhibiting antioxidant enzymes and decreasing glutathione (GSH) levels, ${ }^{29,30}$ which are also the underlying factors of nephrotoxicity. Free radicals oxidize the lipids of cell membrane and alters membrane permeability, leading to excessive calcium influx and mitochondrial dysfunction. Impaired mitochondrial function in the renal proximal tubule epithelial cells affects the reabsorption of glucose, resulting in edema and cellular degeneration. ${ }^{30}$ Compared to the untreated controls, cisplatin significantly reduced the content of the antioxidant enzyme GSH as well as the activity of SOD in renal tissues, and increased the levels of the lipid peroxidation product MDA. Thus, cisplatin treatment impaired the ability of renal cells to scavenge the free radicals, which was restored by raloxifene, in agreement with its previously reported antioxidant effects. Interestingly, the indices of oxidative damage were not affected in the liver tissues of cisplatin-treated mice, indicating that the protective effect of raloxifene on cisplatininduced liver injury involves other mechanisms.

Various antioxidants have been applied to inhibit oxidative stress of cisplatin-induced nephrotoxicity, such as amifostine N-acetylcysteine (NAC), Superoxide dismutase (SOD) and catalase (CAT). However, although several kinds of drugs are applied clinically in response to cisplatininduced kidney injury, these drugs exhibit different degrees of shortage. Amifostine is a broad-spectrum cytoprotective agent approved by the FDA as a nephroprotective agent during cisplatin chemotherapy in patients with advanced ovarian cancer; however, due to blood pressure drops and hypocalcemia, its application in other tumors is limited. ${ }^{31,32}$ $\mathrm{N}$-acetylcysteine (NAC) is widely used to treat paracetamol overdose, with a remarkable antioxidant and antiinflammatory capacity. Javaherforooshzadeh $\mathrm{F}$ found that administration of $\mathrm{N}$-acetylcysteine can reduce the incidence of acute kidney injury in patients treated coronary artery bypass graft surgery and promoted kidney functions. ${ }^{33}$ However, another study indicated that when evaluated in patients with stage 3 chronic kidney disease, $600 \mathrm{mg}$ oral NAC supplementation was not able to alter serum creatinine or Cystatin C. ${ }^{34}$ Lastly, there is the debate regarding NAC efficacy with kidney disease. Both SOD and CAT are antioxidant enzyme in the body. SOD acts as a catalyzes the dismutation of superoxide. The reaction helps to conduit in transmission of the injury caused by free radicals but with hydrogen peroxide as a by-product. CAT acts as a catalyst for the conversion of hydrogen peroxide to oxygen and water. Both SOD and CAT plays an important role in antiaging and disease treatment. However, because of extremely rapid plasma clearance time and poor stability, the clinical application of SOD and CAT as therapeutic agents was very limited. ${ }^{35-37}$ By comparison, raloxifene with a good stability and administration is highly tolerated and does not present significant adverse effects. It was well tolerated with doses of $30-600 \mathrm{mg} /$ day in clinical trials which the subjects most were postmenopausal women. The most common side-effects were hot flushes and leg cramps. $^{38,39}$

There are several studies have shown that raloxifene can induce apoptosis on tumor cells and reduces endometrial cancer risk in women. Shi W demonstrated raloxifene inhibited constitutive activation of STAT3 in breast cancer cell-line MDB-MB-231, colon cancer cell-line HCT116 and multiple myeloma cancer cell-line U266. Raloxifene also inhibited phosphorylation of STAT3 induced by IL-6 in MCF-7, HT29 and MM.1S cancer cell lines. ${ }^{40}$ DeMichele A provides evidence that raloxifene reduces endometrial cancer risk in women with a populationbased, case-control study. ${ }^{7}$ Olivier $\mathrm{S}$ found raloxifene induces myeloma cell cycle arrest and apoptosis through NF-kappa B-dependent mechanisms. ${ }^{41}$ Jiang HS reported raloxifene induces apoptosis in estrogen receptor-negative human cancer cells through the aryl hydrocarbon receptor $(\mathrm{AhR}){ }^{42}$ Based on these evidences, we speculate that raloxifene may enhance the therapeutic effect of cisplatin on tumors. 


\section{Findings and Limitations}

Some findings of this study have not been reported in the past: In this study, we demonstrated for the first time that pretreatment with raloxifene protected against cisplatininduced acute kidney injury through inhibition of oxidative stress. But, there are some limitations in this study. We have preliminarily studied and proved raloxifene protected against cisplatin-induced acute kidney injury by histopathological and biochemical analyses, but we have not explored it very deeply, especially molecular mechanism. In addition, although many biological functions and behavior of mice are close to human and mice are good models for study, our results cannot completely represent the effects on human. Clinical trial needs to be conducted to elucidate raloxifene to protect against cisplatin-induced nephrotoxicity.

\section{Conclusion}

Raloxifene alleviates cisplatin-induced acute renal injury by reversing oxidative stress, and has a protective effect on the liver as well. Raloxifene can reduce the side effects of cisplatin when used as an adjuvant. Our findings also indicate that a drug-drug interaction might occur with coadministration of raloxifene and cisplatin, which could affect the cisplatin content and distribution in the kidney and in the HK-2 cells. We suggest that raloxifene used in combination with cisplatin during chemotherapy, which is of great clinical significance. As raloxifene showed no toxic effects in HK-2 cells and mice, we propose that raloxifene should be considered as a nephroprotective agent during cisplatin chemotherapy.

\section{Acknowledgments}

We are grateful to Dr. Ji-Yan Chen, at the Department of Cardiology, Guangdong Provincial people's Hospital affiliated to South China University of Technology for providing HK-2 cell lines. We are grateful to Dr. XiaoQin Zhang, at the School of Medicine, South China University of Technology for her fund support. This work was supported by grants from research funding for part-time professors of South China University of Technology (No. D6202140) and the National Natural Science Foundation of China (No.81702945). We thank Xiao-Tao Hou (Guangzhou KingMed Diagnostics Group Co., Ltd.) and Jiao Luo (Renal Division, Nanfang Hospital, Southern Medical University) for expert assistance with TEM preparation and image acquisition.

\section{Disclosure}

The authors have declared that no competing interest exists.

\section{References}

1. Ghosh S. Cisplatin: the first metal based anticancer drug. Bioorg Chem. 2019;88:102925. doi:10.1016/j.bioorg.2019.102925

2. National Cancer Institute. Drug Dictionary: cisplatin; 2021. Available from: https://www.cancer.gov/publications/dictionaries/cancer-drug /def/cisplatin. Accessed August 8, 2021.

3. Dasari S, Tchounwou PB. Cisplatin in cancer therapy: molecular mechanisms of action. Eur J Pharmacol. 2014;740(1):364-378. doi:10.1016/j.ejphar.2014.07.025

4. McSweeney KR, Gadanec LK, Qaradakhi T, et al. Mechanisms of cisplatin-induced acute kidney injury: pathological mechanisms, pharmacological interventions, and genetic mitigations. Cancers. 2021;13(7):1572. doi:10.3390/cancers13071572

5. Cocetta V, Ragazzi E, Montopoli M. Mitochondrial involvement in cisplatin resistance. Int J Mol Sci. 2019;20(14):3384. doi:10.3390/ ijms 20143384

6. Goldstein SR. Selective estrogen receptor modulators and bone health. Climacteric. 2021. doi:10.1080/13697137.2021.1936485

7. DeMichele A, Troxel AB, Berlin JA, et al. Impact of raloxifene or tamoxifen use on endometrial cancer risk: a population-based case-control study. J Clin Oncol. 2008;26(25):4151-4159. doi:10. 1200/JCO.2007.14.0921

8. Osmanova S, Sezer E, Turan V, et al. The effects of raloxifene treatment on oxidative status in brain tissues and learning process of ovariectomized rats. Iran J Reprod Med. 2011;9(4):295-300.

9. Yazğan B, Yazğan Y, Övey İS, et al. Raloxifene and tamoxifen reduce PARP activity, cytokine and oxidative stress levels in the brain and blood of ovariectomized rats. J Mol Neurosci. 2016;60(2):214-222. doi:10.1007/s12031-016-0785-9

10. Vesga-Jiménez DJ, Hidalgo-Lanussa O, Baez-Jurado E, et al. Raloxifene attenuates oxidative stress and preserves mitochondrial function in astrocytic cells upon glucose deprivation. J Cell Physiol. 2019;234(3):2051-2057. doi:10.1002/jcp.27481

11. Wassmann S, Laufs U, Stamenkovic D, et al. Raloxifene improves endothelial dysfunction in hypertension by reduced oxidative stress and enhanced nitric oxide production. Circulation. 2002;105 (17):2083-2091. doi:10.1161/01.CIR.0000014618.91633.67

12. Sun Q, Nan XY, Tian FM, et al. Raloxifene retards the progression of adjacent segmental intervertebral disc degeneration by inhibiting apoptosis of nucleus pulposus in ovariectomized rats. J. Orthop Surg Res. 2021;16(1):368. doi:10.1186/s13018-021-02504-4

13. Chiou WC, Hsu MS, Chen YT, et al. Repurposing existing drugs: identification of SARS-CoV-2 3C-like protease inhibitors. J Enzyme Inhib Med Chem. 2021;36(1):147-153. doi:10.1080/14756366.20 20.1850710

14. Hong S, Chang J, Jeong K, et al. Raloxifene as a treatment option for viral infections. J Microbiol. 2021;59(2):124-131. doi:10.1007/ s12275-021-0617-7

15. Siegel RL, Miller KD, Fuchs HE, et al. Cancer statistics, 2021. $C A$ Cancer J Clin. 2021;71(1):7-33. doi:10.3322/caac.21654

16. Florea AM, Büsselberg D. Cisplatin as an anti-tumor drug: cellular mechanisms of activity, drug resistance and induced side effects. Cancers. 2011;3(1):1351-1371. doi:10.3390/cancers3011351

17. Kollmannsberger C, Honecker F, Bokemeyer C. Pharmacotherapy of relapsed metastatic testicular cancer. Expert Opin Pharmacother. 2008;9(13):2259-2272. doi:10.1517/14656566.9.13.2259

18. Razzaque MS. Cisplatin nephropathy: is cytotoxicity avoidable? Nephrol Dial Transplant. 2007;22(8):2112-2116. doi:10.1093/ndt/ gfm 378 
19. Safirstein R, Winston J, Goldstein M, et al. Cisplatin nephrotoxicity. Am J Kidney Dis. 1986;8(5):356-367. doi:10.1016/S0272-6386(86)80111-1

20. Filipski KK, Mathijssen RH, Mikkelsen TS, et al. Contribution of organic cation transporter 2 (OCT2) to cisplatin-induced nephrotoxicity. Clin Pharmacol Ther. 2009;86(4):396-402. doi:10.1038/clpt.2009.139

21. Ozkok A, Edelstein CL. Pathophysiology of cisplatin-induced acute kidney injury. Biomed Res Int. 2014;2014:967826. doi:10.1155/2014/967826

22. Abd El-Rhman RH, El-Naga RN, Gad AM, et al. Dibenzazepine attenuates against cisplatin-induced nephrotoxicity in rats: involvement of NOTCH pathway. Front Pharmacol. 2020;11:567852. doi:10.3389/fphar.2020.567852

23. Ko JW, Shin NR, Jung TY, et al. Melatonin attenuates cisplatin-induced acute kidney injury in rats via induction of anti-aging protein, Klotho. Food Chem Toxicol. 2019;129:201-210. doi:10.1016/j.fct.2019.04.049

24. Kandemir FM, Yildirim S, Caglayan C, et al. Protective effects of zingerone on cisplatin-induced nephrotoxicity in female rats. Environ Sci Pollut Res Int. 2019;26(22):22562-22574. doi:10.1007/s11356019-05505-3

25. Abdellatief SA, Galal AA, Farouk SM, et al. Ameliorative effect of parsley oil on cisplatin-induced hepato-cardiotoxicity: a biochemical, histopathological, and immunohistochemical study. Biomed Pharmacother. 2017;86:482-491. doi:10.1016/j.biopha.2016.12.038

26. Abdel-Daim MM, Abdel-Rahman HG, Dessouki AA, et al. Impact of garlic (Allium sativum) oil on cisplatin-induced hepatorenal biochemical and histopathological alterations in rats. Sci Total Environ. 2020;710:136338. doi:10.1016/j.scitotenv.2019.136338

27. Chirino YI, Pedraza-Chaverri J. Role of oxidative and nitrosative stress in cisplatin-induced nephrotoxicity. Exp Toxicol Pathol. 2009;61(3):223-242. doi:10.1016/j.etp.2008.09.003

28. Rodriguez E, Pei G, Kim ST, et al. Substance P antagonism as a novel therapeutic option to enhance efficacy of cisplatin in triple negative breast cancer and protect PC12 cells against cisplatin-induced oxidative stress and apoptosis. Cancers. 2021;13 (15):3871. doi:10.3390/cancers 13153871

29. Sadzuka Y, Shoji T, Takino Y. Mechanism of the increase in lipid peroxide induced by cisplatin in the kidneys of rats. Toxicol Lett. 1992;62(2-3):293-300. doi:10.1016/0378-4274(92)90033-G

30. Zhang JG, Lindup WE. Role of mitochondria in cisplatin-induced oxidative damage exhibited by rat renal cortical slices. Biochem Pharmacol. 1993;45(11):2215-2222. doi:10.1016/0006-2952(93)90192-Y
31. Capizzi RL. Amifostine reduces the incidence of cumulative nephrotoxicity from cisplatin: laboratory and clinical aspects. Semin Oncol. 1999;26(2 Suppl 7):72-81.

32. Culy CR, Spencer CM. Amifostine. Drugs. 2001;61(5):641-684. doi:10.2165/00003495-200161050-00012

33. Javaherforooshzadeh F, Shaker Z, Rashidi M, et al. The effect of $\mathrm{N}$-acetyl cysteine injection on renal function after coronary artery bypass graft surgery: a randomized double blind clinical trial. J Cardiothorac Surg. 2021;16(1):161. doi:10.1186/s13019-02101550-7

34. Mainra R, Gallo K, Moist L. Effect of N-acetylcysteine on renal function in patients with chronic kidney disease. Nephrology. 2007;12(5):510-513. doi:10.1111/j.1440-1797.2007.00833.x

35. Porfire AS, Leucuţa SE, Kiss B, et al. Investigation into the role of $\mathrm{Cu} / \mathrm{Zn}$-SOD delivery system on its antioxidant and antiinflammatory activity in rat model of peritonitis. Pharmacol Rep. 2014;66 (4):670-676. doi:10.1016/j.pharep.2014.03.011

36. Jeeva JS, Sunitha J, Ananthalakshmi R, et al. Enzymatic antioxidants and its role in oral diseases. J Pharm Bioallied Sci. 2015;7(Suppl 2): S331-S333. doi:10.4103/0975-7406.163438

37. Younus H. Therapeutic potentials of superoxide dismutase. Int J Health Sci. 2018;12(3):88-93.

38. Goldstein SR, Siddhanti S, Ciaccia AV, et al. A pharmacological review of selective oestrogen receptor modulators. Hum Reprod Update. 2000;6(3):212-224. doi:10.1093/humupd/6.3.212

39. Lu Y, Cohen F, Lakshmanan M. Characterization of hot flashes during raloxifene therapy: analyses of adverse event reports from an integrated placebo-controlled clinical trial database. Menopause. 1998;5(4):282. doi:10.1097/00042192-199805040-00136

40. Shi W, Yan D, Zhao C, et al. Inhibition of IL-6/STAT3 signaling in human cancer cells using Evista. Biochem Biophys Res Commun. 2017;491(1):159-165. doi:10.1016/j.bbrc.2017.07.067

41. Olivier S, Close P, Castermans E, et al. Raloxifene-induced myeloma cell apoptosis: a study of nuclear factor-kappa B inhibition and gene expression signature. Mol Pharmacol. 2006;69(5):1615-1623. doi:10.1124/mol.105.020479

42. Jang HS, Pearce M, O'Donnell EF, et al. Identification of a raloxifene analog that promotes AhR-mediated apoptosis in cancer cells. Biology. 2017;6(4):41. doi:10.3390/biology6040041
OncoTargets and Therapy

\section{Publish your work in this journal}

OncoTargets and Therapy is an international, peer-reviewed, open access journal focusing on the pathological basis of all cancers, potential targets for therapy and treatment protocols employed to improve the management of cancer patients. The journal also focuses on the impact of management programs and new therapeutic agents and protocols on patient perspectives such as quality of life, adherence and satisfaction. The manuscript management system is completely online and includes a very quick and fair peer-review system, which is all easy to use. Visit http://www.dovepress.com/ testimonials.php to read real quotes from published authors. 